\title{
Maternal Outcome in Mitral Valve Disease with Pulmonary Hypertension: Two Case Reports
}

\author{
Anuradha Alagandala ${ }^{1} \quad$ Daya Vaswani $\quad$ Vuduthala Trinath Bharadwaj ${ }^{3} \quad$ Kousalya Chakravarthy $^{1}$ \\ ${ }^{1}$ Department of Anaesthesia, MGMH Petlaburj, Osmania Medical \\ College, Hyderabad, Telangana, India \\ 2Department of Cardiology, NIMS, Hyderabad, Telangana, India \\ Address for correspondence Kousalya Chakravarthy, MD, DGO, \\ 3-5-1083, Flat 306, Sri Tarajeet Residency, Narayanguda 500029, \\ Hyderabad, Telangana, India (e-mail:dr.kausi.c@gmail.com).
}

${ }^{3}$ Osmania Medical College, Hyderabad, Telangana, India

\begin{abstract}
Keywords

- cesarean delivery

- mitral stenosis

- pulmonary embolism

- pregnancy

Chronic rheumatic heart disease is still the leading cause of heart disease complicating pregnancy in the developing countries. The physiologic changes in pregnancy and stress induced by the increase in cardiac output can cause asymptomatic patients with mitral stenosis to decompensate, especially in the third trimester. Severity of mitral stenosis is reflected by the decrease in the valve area and increase in the right ventricular systolic pressures (RVSPs). Venous thromboembolism (VTE) is two to five times more common in the postpartum period, and the risk is high with cesarean delivery. Pulmonary embolism in pregnancy can cause severe maternal morbidity and mortality. We describe two cases of severe mitral stenosis complicating pregnancy with grossly elevated RVSP. The first case is the successful management of a second gravida, presenting with severe mitral stenosis with RVSP $80 \mathrm{~mm} \mathrm{Hg}$, and the second, a fatal case of a 26-year-old para 1, live 1, delivered by cesarean section, presenting with massive fatal pulmonary embolism on 13th postoperative day (POD) with RVSP $90 \mathrm{~mm}$ $\mathrm{Hg}$. A multidisciplinary approach involving the cardiologist, obstetrician, and obstetric anesthesiologist is crucial for management of severe mitral stenosis complicating pregnancy, to decrease the maternal morbidity and mortality during peripartum period.
\end{abstract}

\section{Introduction}

Mitral stenosis (MS) is the most common cause of chronic rheumatic heart disease (CRHD) complicating pregnancy. ${ }^{1}$ Pulmonary edema and cardiac decompensation can occur at any time of pregnancy, depending on the severity of the lesion and the associated complications. Severity of MS is reflected by the decrease in the valve area and increase in right ventricular pressures. Symptomatic severe isolated MS in pregnancy with pulmonary arterial hypertension (PAH) can be managed by percutaneous balloon mitral valvuloplasty (PBMV), ideally in the second trimester, but it can be done at any time of pregnancy. Severe MS with mitral regurgitation (MR) mandates mitral valve surgery that carries a high maternal fetal morbidity and mortality. ${ }^{2}$ Venous thromboembolism (VTE) is two to five times more common in the postpartum, and the risk is higher with cesarean delivery. The added risk of postpartum thromboembolism predisposes pregnant women with severe MS to comorbidities such as pulmonary embolism (PE), thus increasing the maternal morbidity and mortality.

We report the maternal outcome of two cases of severe MS with severe PAH, with grossly elevated right ventricular systolic pressure (RVSP), in which maternal mortality and morbidity are high as isolated valvular lesion itself. In addition, the first case had significant MR and the second case presented with massive PE.

\section{Case Report 1}

A 27-year-old gravida 4, para 1, live 1, and abortion 2 presented to our hospital at 36 weeks of gestational age with acute breathlessness. The patient had a similar complaint in her last pregnancy. History revealed that she delivered 18 months back by lower segment cesarean section (LSCS) under spinal anesthesia. On the second postoperative day 
(POD), the patient developed shortness of breath (SOB) and hemoptysis for which she was evaluated by two-dimensional (2D) echo, which revealed severe MS and severe PAH. She was referred to cardiology intensive care unit (ICU) and was evaluated for valve replacement. As a part of evaluation, transesophageal echo (TEE) was done. TEE confirmed severe MS, severe PAH, significant MR, a subvalvular prolapse (probably significant subvalvular pathology precluding percutaneous balloon mitral valvoplasty [PBMV]), mild aortic regurgitation (AR) and tricuspid regurgitation (TR), and organic tricuspid valve (TV) with normal biventricular systolic pressures. The patient was advised mitral valve replacement and was advised against further pregnancies.

Nevertheless, the patient conceived and presented at 36 weeks of gestation with history of dyspnea and palpitations and orthopnea since 3 months. Echo on admission revealed severe MS, mitral valve area (MVA) of $1 \mathrm{~cm}^{2}$, severe PAH with RVSP of $85 \mathrm{~mm} \mathrm{Hg}$ and significant MR, mild TR and organic TV, dilated left atrium (LA), no regional wall motion abnormality (RWMA), and good left ventricular/right ventricular (LV/RV) function. Preoperative optimization was done with rest, intravenous furosemide $40 \mathrm{mg}$ 8th hourly and metoprolol $12.5 \mathrm{mg}$ 12th hourly for 1 week. Two-dimensional (2D) echo repeated again after preoperative optimization showed RVSP reduced to $60 \mathrm{~mm} \mathrm{Hg}$ and mitral valve gradient (MVG) $40 / 35 \mathrm{~mm} \mathrm{Hg}$, significant MR, thickened aortic valve (AV), mild TR, organic TV, dilated LA $4.1 \mathrm{~cm}^{2}$, no RWMA, and good LV/RV function. Risk factors for this patient included New York Heart Association (NYHA) class III-IV, CARPREG (cardiac lesions in pregnancy $)^{3}$ score $>1$, RVSP 85 to $60 \mathrm{~mm} \mathrm{Hg}$, dilated LA $4.1 \mathrm{~cm}^{2}$, and previous LSCS. After weighing the pros and cons of general versus regional anesthesia, the patient was taken up for LSCS, incremental epidural dose was given, and hemodynamics were monitored with left radial artery and right internal jugular vein (IJV) cannulation. Phenylephrine was given as a prophylactic vasopressor. A healthy baby with Apgar 8,9 was delivered. Post-delivery, frusemide $40 \mathrm{mg}$ bolus was given to combat autotransfusion. Concentrated oxytocin infusion at a rate of $5 \mathrm{U} / \mathrm{h}$ was given to restrict the fluid overload and oxytocin-induced tachycardia. Intraoperative period was uneventful.

One hour into postoperative period, the patient complained of SOB. There were peripheral cyanosis, fall in blood pressure, and saturations suggestive of cardiac failure. Inotropic support with noradrenaline started, and the patient was put on intermittent noninvasive ventilation (NIV). Loop diuretic frusemide $40 \mathrm{mg}$ was given every 8 hours to decrease the preload, and oral metoprolol $12.5 \mathrm{mg}$ continued for rate control. Postoperative analgesia was given through epidural catheter. Hemodynamic stability was achieved within 1 hour. Early oral liquids started 5 hours after the surgery. Thromboprophylaxis with subcutaneous low-molecular-weight heparin (LMWH) enoxaparin $40 \mathrm{mg}$ started 12 hours after surgery and continued for 1 week. Both the mother and child were discharged in stable condition, with the advice to follow cardiology consultation for mitral valve replacement.

\section{Case Report 2}

A 26-year-old para 1, live 1, delivered by cesarean section (CS), presented with severe SOB on 13th POD. History revealed that the patient was known case of severe MS of rheumatic origin, and she underwent emergency CS for fetal indicationintrauterine growth restriction (IUGR). At the time of presentation, she was drowsy but arousable, acidotic breathing, and pitting pedal edema in both the legs. Blood pressure was not recordable at time of arrival. Pulse rate was 128 beats/min regular, respiratory rate was 46 breaths/min, and saturation was $60 \%$ on room air that improved to $100 \%$ on facemask with $10 \mathrm{~L}$ of oxygen. Arterial blood gas analysis with oxygen mask revealed severe hypoxia and acidotic state with $\mathrm{pH}$ 7.046, $\mathrm{PO}_{2}$ $159, \mathrm{PCO}_{2}$ 17.9, $\mathrm{HCO}_{3} 4.8$, anion gap 25.8, and lactate 15.64 . Previous reports showed $\mathrm{Hb} 13.1 \mathrm{~g} \%$. Total leukocyte count was 16,700 , platelet count $190,000 / \mathrm{mm}^{3}$, blood urea $76 \mathrm{mg} \%$, and serum creatine $1.8 \mathrm{mg} / \mathrm{dL}$. Echo done before cesarean delivery showed severe MS with TR, moderate PAH, mild MR, ejection fraction (EF) 60\%, and normal LV function.

\section{Investigations}

Electrocardiogram (ECG) showed sinus tachycardia ( - Fig. 1), and anteroposterior (AP) view of chest $X$ ray was suggestive of pulmonary edema ( - Fig. 2). Two-dimensional echo showed dilated LA, dilated right atrium (RA) and right ventricle $(\mathrm{RV})$, dilated pulmonary artery, severe MS with MVA $0.9 \mathrm{~cm}^{2}$, MVG 21/16 mm $\mathrm{Hg}$, trivial MR, no aortic stenosis (AS) or AR, severe TR, severe PAH with RVSP $90 \mathrm{~mm} \mathrm{Hg}$, grade I diastolic dysfunction, good RV function, and no PE/veg/clot but suspicion of the thrombus at the distal bifurcation of the pulmonary artery. Lactate dehydrogenase (LDH) was 1,450, and fibrin degradation products (FDPs) were strongly positive (only quantitative test was done). Dilated RA, RV, and pulmonary artery, and increased LDH and FDP in the setting of severe MS with RVSP $90 \mathrm{~mm}$ Hg supported the diagnosis of massive PE. The increase in severity of PAH prompted the authors to consider other causes of PAH such as pulmonary thromboembolism. Further investigations were precluded in view of hemodynamic instability.

The final diagnosis was primipara on 13th POD with severe MS in failure, cardiogenic shock with super added massive pulmonary thromboembolism. The patient was put on ventilator support, and medical management of cardiogenic shock started. In view of the hemodynamically unstable condition and prerenal azotemia, the option of thrombectomy or pushing the thrombus into belly by using the pigtail was contemplated, but consent was not given by the patient's attenders. After explaining the benefits and risks to the attenders, thrombolysis was initiated with tissue plasminogen activator (TPA) through the right femoral vein. Despite all the efforts, the patient's condition deteriorated rapidly and she succumbed after 5 hours.

\section{Discussion}

Mitral stenosis of rheumatic origin contributes to $88 \%$ of heart diseases complicating pregnancy in tertiary referral centers 


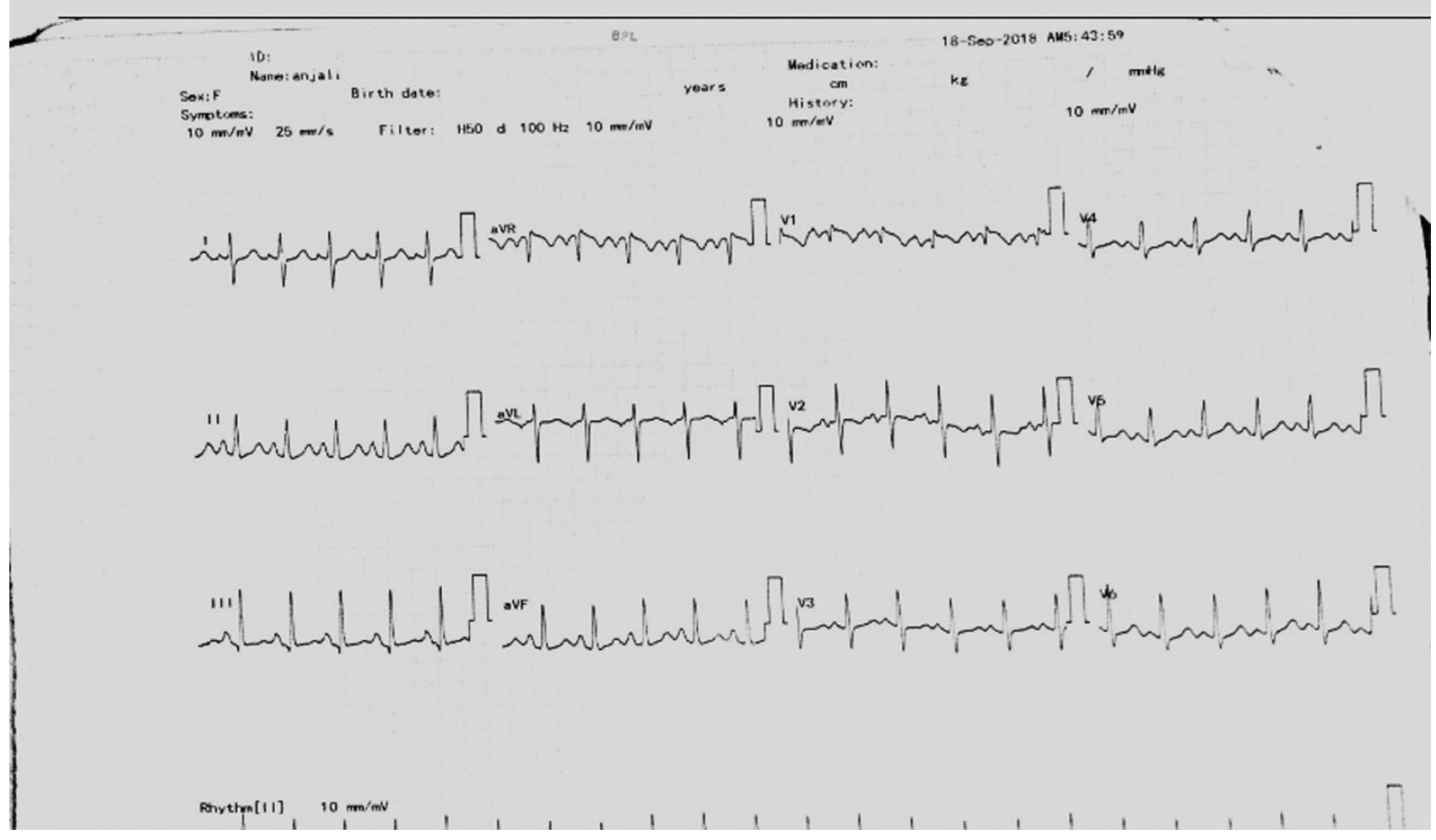

Fig. 1 ECG showing sinus tachycardia.

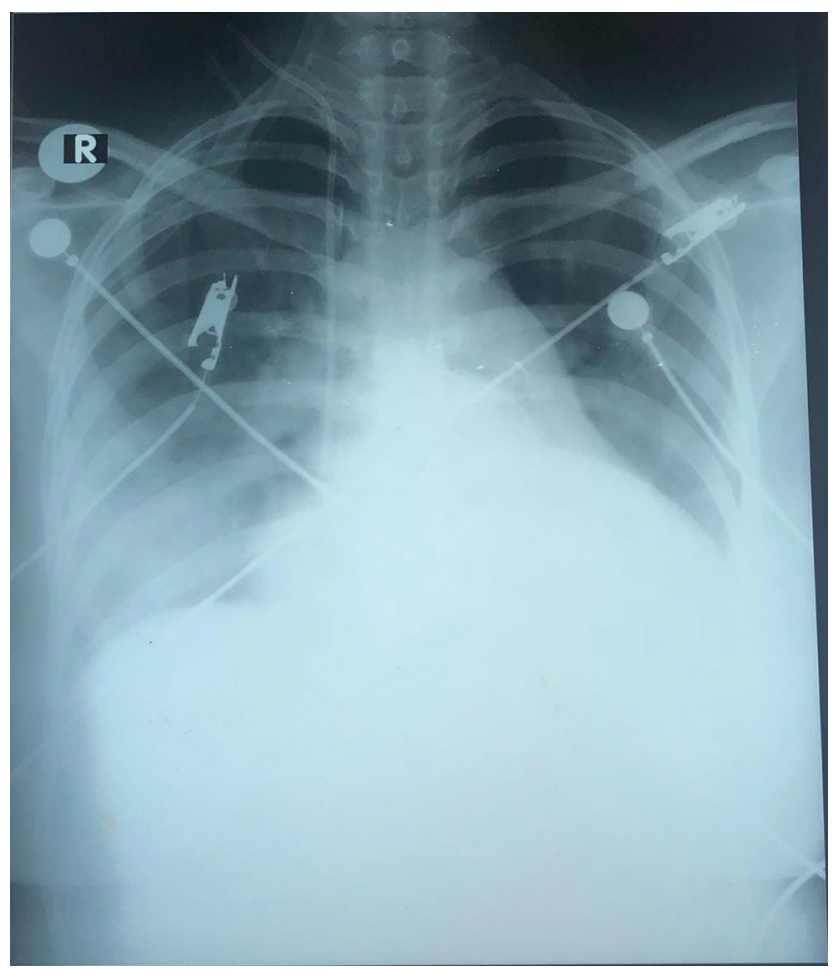

Fig. 2 Chest X ray AP view suggestive of pulmonary edema.

in India. ${ }^{4}$ Stress induced by the increase in cardiac output can cause asymptomatic patients with MS to decompensate during the latter half of pregnancy ${ }^{5}$ presenting as pulmonary edema in $25 \%$ of cases. ${ }^{6}$ The CARPREG $^{3,7}$ score identifies four predictors of major adverse cardiac event: (1) poor functional class (NYHA class II-IV), (2) previous cardiac event (heart failure, transient ischemic attack, stroke) or arrhythmia, (3) left heart obstruction (MVA of $<2 \mathrm{~cm}$, AV area of $<1.5 \mathrm{~cm}$, peak left ventricular outflow gradient $>30 \mathrm{~mm} \mathrm{Hg}$ ), and (4) left ventricular systolic dysfunction (left ventricular $\mathrm{EF}<40 \%$ ). One point is assigned for each finding, and the summed score estimates the cardiac risk. Zero points imply 5\% risk, 1 point carries $27 \%$, and more than 1 point has $75 \%$ risk of major cardiac events. The associated perioperative risks include functional class deterioration, heart failure/cardiogenic shock, and pulmonary edema. The elevated baseline RVSPs by echo reflects the severity of pulmonary involvement and adverse outcome. Selection of anesthesia technique, intensive hemodynamic monitoring, and slow and cautious titration of oxytocic drugs are important in decreasing the perioperative fetomaternal morbidity and mortality. An incremental and controlled dose of epidural neuraxial anesthesia prevents sudden hypotension and provides good hemodynamic stability in heart disease complicating pregnancy. ${ }^{8}$ A working epidural gives good postoperative pain relief, and there is documented decrease in the postoperative pulmonary complications. In our first case, an incremental epidural was given. Phenylephrine as a prophylactic vasopressor effectively prevents hypotension and tachycardia of sympathetic block. ${ }^{9}$ Intraoperative monitoring should be continued in the postoperative period to facilitate early detection of deterioration. Combined valvular lesions such as MS with MR are not amenable to percutaneous interventions. It is better to educate the patient before planning for pregnancy itself to decrease the maternal mortality. In our case, despite the advice against further pregnancy, the patient conceived. Preoperative optimization and meticulous perioperative monitoring helped in successful management of the case. 
Severe MS can cause left atrial dilatation, stasis, and can lead to atrial fibrillation/supraventricular tachycardia (SVT), thrombus formation, and transient ischemic attack (TIA)/ stroke. ${ }^{10}$ The hypercoagulable state makes pregnancy itself a risk factor for VTE, with a prevalence of 1 in $1,600,{ }^{11} 4$ to 50 times higher compared with nonpregnant women. ${ }^{12,13}$ VTE is two to five times more common in the postpartum, ${ }^{14}$ with the risk increased up to 6 weeks after delivery. ${ }^{15}$ Factors that increase the risk of VTE postpartum are CS, especially when emergency, medical comorbidities (e.g., varicose veins, cardiac disease, inflammatory bowel disease). ${ }^{16,17}$ Untreated deep vein thrombosis (DVT) can cause PE in up to $24 \%$ of patients, with an associated mortality rate of approximately $15 \% .{ }^{18} \mathrm{PE}$ is the seventh leading cause of maternal mortality. ${ }^{19}$ Preventing deaths from acute PE in pregnancy needs a high index of clinical suspicion and an accurate diagnostic approach. ${ }^{20}$ In our case, the patient's deterioration can be explained by severe MS, but the clinical presentation with cardiogenic shock on the 13th day postpartum, increased severity of PAH, and omitting of anticoagulation prophylaxis after CS made us suspect super added PE.

The data on the use of thrombolytic drugs for pregnant patients with massive PE are limited..$^{21}$ TPA or recombinant TPA (rtPA) is preferred as it is widely available, has a lower complication profile, can be given over a short time period, and rtPA's mechanism of action is ideal for acute thrombotic events where the pathologic clot can be dissolved without inducing a systemic "lytic" effect. ${ }^{22}$

It has been shown that rtPA has high affinity for plasminogen only in the presence of fibrin, thus affording this theoretical benefit of less systemic hemorrhage risk. Compared to other thrombolytics, rtPA is purported not to induce antigenicity, thus allowing for repeated administration, which is beneficial in the cases of mechanical valve thrombosis. ${ }^{23,24}$ The use of thrombolytics agents in pregnant patients may cause life-threatening hemorrhage (ante- and postpartum) and surgical site bleed in postoperative patients. We had to select this mode of therapy as the choice by the patient's attendants, not as the best modality of therapy.

Cardiovascular collapse associated with a massive pulmonary embolus during pregnancy requires immediate aggressive intervention to save the mother and fetus. Pick et al described successful management of the use of catheter infusion thrombolysis in a third-trimester pregnant patient with a hemodynamically significant saddle pulmonary embolus. ${ }^{25}$ O'Keeffe et al described the use of mechanical fragmentation and optional retrievable inferior vena cava (IVC) filter insertion in a 38-week pregnant woman, followed by delayed pharmacological catheterdirected thrombolysis during the early postpartum period to successfully treat a massive PE. ${ }^{26}$ Pharmacoinvasive therapy was offered to our patient, but consent was not given by the attenders.

A multidisciplinary involvement of the cardiologist, obstetrician, obstetric anesthesiologist, appropriate preoperative optimization, awareness of the perioperative complications, and early management has led to the successful management of our first case of severe MS with PAH and failure. In our second case, the patient reported with massive PE and grossly elevated RVSP and in shock. We started TPA but could not prevent the maternal mortality. Perioperative mechanical thromboprophylaxis with intermittent pneumatic compression (IPC) devices, pharmacologic thromboprophylaxis with enoxaparin $40 \mathrm{mg}$ subcutaneously once a day for 5 to 7 days or until the patient is fully mobile, might have prevented the extensive thrombus formation. Awareness of the condition and an early referral to a cardiac center could have prevented the mortality.

\section{Conclusion}

A prepregnancy counselling, and a proper planning of the course of pregnancy, delivery, and postpartum, is essential to decrease the maternal morbidity in MS complicating pregnancy. A multidisciplinary approach involving a cardiologist, obstetrician, obstetric anesthesiologist, and a neonatologist gives a better maternal and fetal outcome in these cases.

\section{Conflicts of Interest}

None.

\section{Center of Study}

Department of Anaesthesia, MGMH Petlaburj, Osmania Medical College, Hyderabad.

Department of Cardiology, Nizams Institute of Medical Sciences (NIMS), Hyderabad.

\section{Source(s) of Support}

None.

\section{Acknowledgement}

None.

\section{References}

1 Nataraj MS, Giri V. Anesthetic management of cesarean section with mitral stenosis and respiratory tract infection. J Obstet Anaesth Crit Care 2014;4:78-80

2 de Souza JA, Martinez EE Jr, Ambrose JA, et al. Percutaneous balloon mitral valvuloplasty in comparison with open mitral valve commissurotomy for mitral stenosis during pregnancy. EE Am Coll Cardiol 2001;37(3):900-903

3 Jastrow N, Meyer P, Khairy P, et al. Prediction of complications in pregnant women with cardiac diseases referred to a tertiary center. Int J Cardiol 2011;151(2):209-213

4 Bhatla N, Lal S, Behera G, et al. Cardiac disease in pregnancy. Int J Gynaecol Obstet 2003;82(2):153-159

5 Sciscione AC, Ivester T, Largoza M, Manley J, Shlossman P, Colmorgen $\mathrm{GH}$. Acute pulmonary edema in pregnancy. Obstet Gynecol 2003;101(3):511-515

6 Martins LC, Freire CM, Capuruçu CA, Nunes MdoC, Rezende CA. Risk prediction of cardiovascular complications in pregnant women with heart disease. Arq Bras Cardiol 2016;106(4):289-296

7 Mentias A, Patel K, Patel H, et al. Effect of pulmonary vascular pressures on long-term outcome in patients with primary mitral regurgitation.J Am Coll Cardiol 2016;67(25):2952-2961

8 Kuczkowski KM. Labor analgesia for the parturient with cardiac disease: what does an obstetrician need to know? Acta Obstet Gynecol Scand 2004;83(3):223-233 
9 Dyer RA, Reed AR, van Dyk D, et al. Hemodynamic effects of ephedrine, phenylephrine, and the coadministration of phenylephrine with oxytocin during spinal anesthesia for elective cesarean delivery. Anesthesiology 2009;111(4):753-765

10 Kannan M, Vijayanand G. Mitral stenosis and pregnancy: current concepts in anaesthetic practice. Indian J Anaesth 2010;54(5):439-444

11 Morris JM, Algert CS, Roberts CL. Incidence and risk factors for pulmonary embolism in the postpartum period. J Thromb Haemost 2010;8(5):998-1003

12 James AH, Jamison MG, Brancazio LR, Myers ER. Venous thromboembolism during pregnancy and the postpartum period: incidence, risk factors, and mortality. Am J Obstet Gynecol 2006;194(5):1311-1315

13 Bourjeily G, Paidas M, Khalil H, Rosene-Montella K, Rodger M. Pulmonary embolism in pregnancy. Lancet 2010;375(9713):500-512

14 Ghaji N, Boulet SL, Tepper N, Hooper WC. Trends in venous thromboembolism among pregnancy-related hospitalizations, United States, 1994-2009. Am J Obstet Gynecol 2013;209(5):433.e1-433.e8

15 Kamel H, Navi BB, Sriram N, Hovsepian DA, Devereux RB, Elkind MS. Risk of a thrombotic event after the 6-week postpartum period. N Engl J Med 2014;370(14):1307-1315

16 Abdul Sultan A, Grainge MJ, West J, Fleming KM, Nelson-Piercy C, Tata LJ. Impact of risk factors on the timing of first postpartum venous thromboembolism: a population-based cohort study from England. Blood 2014;124(18):2872-2880

17 Blondon M, Casini A, Hoppe KK, Boehlen F, Righini M, Smith NL. Risks of venous thromboembolism after cesarean sections: a meta-analysis. Chest 2016;150(3):572-596

18 Winer-Muram HT, Boone JM, Brown HL, Jennings SG, Mabie WC, Lombardo GT. Pulmonary embolism in pregnant patients: fetal radiation dose with helical CT. Radiology 2002;224(2):487-492
19 Hemlata PG, Tewari S, Chatterjee A. Anaesthetic considerations for balloon mitral valvuloplasty in pregnant patient with severe mitral stenosis: a case report and review of literature. J Clin Diagn Res 2017;11(9):UD01-UD03

20 McClure JH, Cooper GM, Clutton-Brock TH; Centre for Maternal and Child Enquiries. Saving mothers' lives: reviewing maternal deaths to make motherhood safer: 2006-8: a review. Br J Anaesth 2011;107(2):127-132

21 Fasullo S, Maringhini G, Terrazzino G, Ganci F, Paterna S, Di Pasquale P. Thrombolysis for massive pulmonary embolism in pregnancy: a case report. Int J Emerg Med 2011;4:69

22 Loscalzo J, Braunwald E. Tissue plasminogen activator. N Engl J Med 1988;319(14):925-931

23 Gartman EJ. The use of thrombolytic therapy in pregnancy. Obstet Med 2013;6(3):105-111

24 Kanter DS, Mikkola KM, Patel SR, Parker JA, Goldhaber SZ. Thrombolytic therapy for pulmonary embolism. Frequency of intracranial hemorrhage and associated risk factors. Chest 1997;111(5):1241-1245

25 Pick J, Berlin D, Horowitz J, Winokur R, Sista AK, Lichtman AD. Massive pulmonary embolism in pregnancy treated with catheter-directed tissue plasminogen activator. A A Case Rep 2015;4(7):91-94

26 O'Keeffe SA, McGrath A, Ryan JM, Byrne B. Management of a massive pulmonary embolism in a pregnant patient with mechanical fragmentation followed by delayed catheter-directed thrombolysis in the early postpartum period. J Matern Fetal Neonatal Med 2008;21(8):591-594 